\title{
Purine-Requiring Auxotrophs of Coprinus lagopus (sensu Buller)
}

\author{
By D. MOORE \\ The Department of Botany, The University, Hull, and \\ The John Innes Institute, Bayfordbury, Hertfordshire*
}

(Accepted for publication 30 November 1966)

\begin{abstract}
SUMMARY
The allelic relationships between a series of independently-induced adenine mutants of Coprinus lagopus were investigated, and limited complementation maps of three of the loci constructed. By means of nutritional tests and a search for aminoimidazole accumulants in the mycelium the adenine loci were correlated with steps in the purine biosynthetic pathway. Four loci can be assigned to (unknown) steps preceding closure of the imidazole ring, two others to specific steps occurring after closure of the ring. One of these latter represents a deficiency in the enzyme adenylosuccinase.
\end{abstract}

\section{INTRODUCTION}

Approximately 70 independently induced mutants exhibiting a response to adenine have been isolated from wild strains of the Basidiomycete Coprinus lagopus (Day \& Anderson, 1961; D. H. Morgan, personal communication; Moore, 1966; P. R. Day \& C. F. Roberts, personal communication), but only a small proportion have so far been allocated to their respective loci. During the course of mapping work designed to increase the linkage data available for Coprinus lagopus (to be published) it became imperative that such locus allocations should be known. As an extension to this work the relationships of the mutant loci to the different steps in purine biosynthesis were investigated, primarily by a search for aminoimidazole accumulants in the mycelium.

\section{METHODS}

The various strains of Coprinus lagopus used were grown on Petri plates from slope stock cultures, most of the stocks being immediately derived from a collection maintained by D. H. Morgan at the John Innes Institute. Many of these were supplied in the first instance by the workers who originally isolated the mutants (see Table 1). As far as can be ascertained all the mutants here mentioned were isolated following ultraviolet irradiation of wild types, with the exception of strain M38 (ad-1) which is an ethanemethanesulphonate-induced mutant. The methods and media for general culture described by Day (1959) were used. All plate cultures were incubated at $37^{\circ}$.

Complementation tests. The test for functional allelism between different mutants was for growth of their mutual dikaryon on minimal medium. Confrontations of the strains under test were always made on complete medium. After $48-60 \mathrm{hr}$ growth pieces of surface and aerial dikaryotic mycelium were transplanted to plates of complete and minimal media, together with control inocula of the monokaryotic

* Present address, The Department of Botany, The University, Manchester, 13. 
parents. Vigorous growth of the dikaryon on minimal medium showed that the mutants complemented one another, and thus were not allelic. Instances of allelic complementation were rare, only six were observed in a total of 539 tests. Absence of growth on minimal medium indicated that the dikaryon carried allelic mutations. The tests were usually made in triplicate.

Growth tests. Tests for growth of the mutant monokaryons on supplemented minimal media were done on Petri dishes. All supplements were added to a final concentration of $50 \mu \mathrm{g} . / \mathrm{ml}$. Conditions of growth were standardized as far as possible by preparing and autoclaving all media at the same time, using a measured $20 \mathrm{ml}$. of medium in plastic Petri dishes, standard punch-cut inocula, and incubation in a single incubator for the same time period. Colony diameters were averaged from 12 measurements of three replicates. In all but a few cases individual measurements varied from the mean value by not more than $10 \%$.

Aminoimidazole accumulants. Attempts to determine the possible positions in the purine biosynthetic pathway were centred on a search for aminoimidazole accumulants in the mycelium, the methods used being those of Bernstein (1961) with modifications. To obtain the quantities of mycelium necessary for the extraction procedures the strains were grown in liquid culture. Suspensions of oidia prepared from slope cultures were used, unfiltered, as inocula for 21 . growth flasks containing 11 . of complete medium. Flasks were incubated on an orbital shaker at $31^{\circ}$ (temperature determined by other users of the machine). After 3-4 days the mycelial mass was filtered off, washed with sterile distilled water, and resuspended in a starvation medium for a further $48 \mathrm{hr}$. The starvation medium was the normal minimal medium to which casein hydrolysate $(0.1 \%, \mathrm{w} / \mathrm{v})$ was added to increase the total yield of aminoimidazoles (Gollub \& Gots, 1959). Mycelium was finally harvested by filtration through cheesecloth, washed with water, blotted and weighed. The yields of mycelium varied with the vigour of the mutants, but suitable choice of the initial incubation period allowed enough mycelium to be obtained even from the slowest growing strain. Eighteen liquid cultures gave amounts of mycelium varying from 20 to $81 \mathrm{~g}$. (pad wet weights); over-all average, $49 \mathrm{~g}$.

Extraction of mycelium was effected by boiling in distilled water $(200 \mathrm{ml} / 20 \mathrm{~g}$. mycelium) for 1-2 min., followed by disintegration in a Waring Blendor. The extracts, after filtration through Whatman no. 1 filter paper, were lyophilized. Lyophilization left a light friable powder (average weight about $0.65 \mathrm{~g}$.) which was completely taken up in $5 \mathrm{ml}$. of distilled water. Debris was removed by centrifugation; proteins were precipitated by boiling for $15 \mathrm{~min}$. and then centrifuged down. The soluble fraction was then ready for chromatography.

Extracts were spotted on to Whatman no. 1 paper and resolved by descending chromatography in a solvent system of isopropanol + water + ammonia (sp.gr. 0.88); $7+2+1$ by volume. Twelve hr at room temperature gave adequate resolution, and after drying the chromatograms were sprayed lightly with the diazosulphonic acid reagents of Ames \& Mitchell (1952). This technique allowed the detection of several aminoimidazole accumulation products not present in extracts of wild-type strains. Identification of the accumulants was attempted with quantities prepared by chromatographing heavy streaks of the extract concerned. The position of the required band was revealed by spraying strips cut from each side of the dried chromatogram, the band cut out and then eluted in water or buffer. 
Enzymic activity of mycelia. Since the extracts were prepared from cultures which had been subjected to a long period of starvation, while the method depended on continued enzymic activity producing the accumulants, it was thought desirable to have some indication of enzyme activity at the time of harvesting. Accordingly the optimum assay conditions for glutamic dehydrogenase (GDH) were determined; this enzyme was known to be present in Coprinus lagopus (Day, 1962) and assay procedures for Neurospora GDH were available. It was assumed that the activity of this enzyme, which is not directly related to purine biosynthesis, would give some indication of the metabolic status of the starved mycelium. If anything, enzymes of the purine pathway would be expected to be de-repressed and thus to show a higher activity than the measured GDH. The observation that Coprinus lagopus GDH is NAD specific was confirmed (Day, 1962). The assay mixture routinely used comprised: $\mathrm{NH}_{4} \mathrm{Cl}$ (M) $0.1 \mathrm{ml}$; 2-oxoglutarate $(0.1 \mathrm{M}) 0.25 \mathrm{ml}$; $\mathrm{NADH}_{2}(2 \mathrm{mg} . / \mathrm{ml}$.) $0.1 \mathrm{ml}$; tris buffer (pH $8.5,0.05 \mathrm{M}) 2.45$ or $2.50 \mathrm{ml}$; crude enzyme extract 0.10 or $0.05 \mathrm{ml}$. The reaction, at $5^{\circ}$, was followed spectrophotometrically by observing the rate of change of extinction at $340 \mathrm{~m} \mu$. Crude enzyme extracts were prepared by grinding about $1 \mathrm{~g}$. of mycelial pad in $5 \mathrm{ml}$. $0.05 \mathrm{M}$-phosphate buffer ( $\mathrm{pH}$ 8) containing 10-3 M-EDTA. Activities varied from $21 \%$ to $133 \%$ of the activity of extracts of a wild strain subjected to the same treatment. It was concluded that, with an average of $54 \%$ of wild type activity, it was most likely that the enzyme concentrations in all samples were satisfactory.

Abbreviations. The following abbreviations are used (after Bernstein, 1961): AMP, adenosine-5'-phosphate; IMP, inosinic acid; GMP, guanosine-5'-phosphate; AMPS, adenylosuccinic acid ribotide; AICAR, 5-amino-4-imidazolecarboxamide ribotide (or riboside); SAICAR, 5-amino-4-imidazole- $N$-succinocarboxamide ribotide or riboside; CAIR, 5-amino-4-imidazole-carboxylic acid ribotide; AIR, 5-aminoimidazole ribotide or riboside.

\section{RESULTS}

Complementation patterns. The adenine mutants could be allocated by means of the dikaryon complementation test into eleven loci (Table 1), six of which have so far been mapped genetically (Day \& Anderson, 1961; D. Moore, unpublished). The ad-7 locus was designated by P. R. Day; it was represented by a single strain which appears to have been lost from all culture collections. Although there are six alleles of ad-8 no allelic complementation was observed for the pairs that were tested. However, allelic complementation was observed among alleles of $a d-1, a d-2$ and $a d-3$. The limited complementation maps which can be drawn for these three loci are shown in Fig. 1. These maps satisfy all the data to hand, but since these are not yet complete the maps almost certainly represent simplifications of the situation at each locus.

Growth tests and search for aminoimidazoles. All the mutants showed a fair to good response to adenine supplementation, while none responded to guanine, indicating a uniform lack of GMP-reductase which is responsible for the conversion of GMP to IMP (Magasanik \& Karibian, 1960). Guanine inhibited the growth of the wild type by about $60 \%$ when present alone, and completely inhibited growth of the wild type when adenine or adenosine were also present in the medium. The latter observation can be explained on the assumption that exogenous adenine or adenosine represses the de novo synthesis of purines, but that competitive inhibition by guanine prevents the 
$a d-1$

(a)

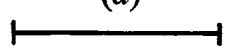

(b)

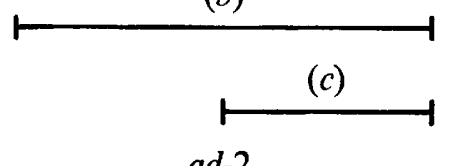

ad-2

(1)

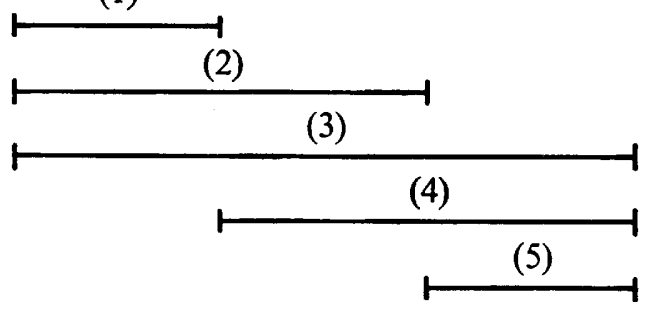

$a d-3$

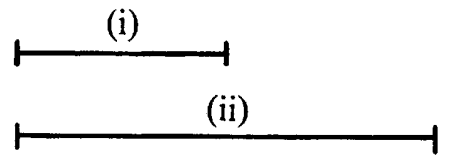

(iii)

Fig. 1. Complementation patterns at three distinct adenine loci. The figures in parentheses indicate the complementation groups which are composed of the following mutants. ad-1: (a) G 1912, (b) с834, (c) м 38. ad-2: (1) G 2302, м63, м64, (2) м 39, (3) м40, Р 816, Р 835, (4) м 58, (5) G 2215, ad-3: (i) M 8, (ii) $\mathrm{C} 691, \mathrm{M} 5, \mathrm{M} 42, \mathrm{M} 65, \mathrm{M} 66, \mathrm{M} 67, \mathrm{M} 68, \mathrm{P} 823, \mathrm{P} 932$, (iii) P 819. The maps were drawn using the established principles for expressing complementation data; i.e. where the bars overlap no complementation was observed between the members of the two groups, where the bars do not overlap complementation was observed. Thus, for example, members of group (1) do not complement each other and do not show complementation with members of groups (2) or (3) (the bars overlap), but they do complement with members of groups (4) and (5).

Table 1. Locus allocations of Coprinus lagopus purine mutants

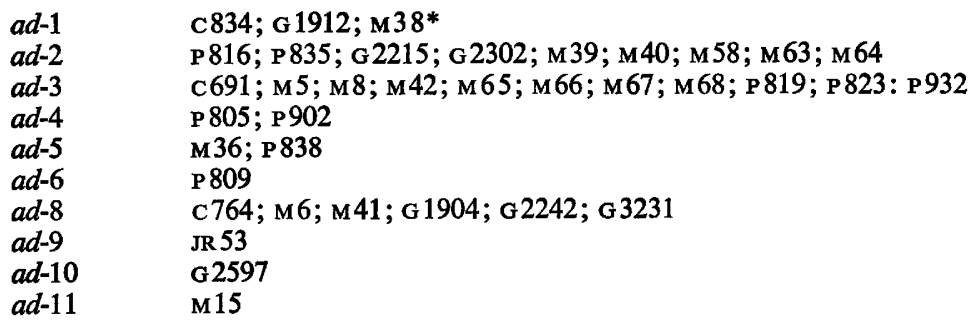

*Stock numbers prefixed by the letter c indicate mutants isolated by D. Lewis; by G, G. E. Anderson; by M, D. H. Morgan (mutants M63 to M70 isolated by the present author); and by $P, P$. R. Day. 
entry of adenine into the organism in sufficient quantity to support growth. Guanosine showed no such inhibition of the wild type whether alone or in company with adenine or adenosine. Table 2 summarizes the response patterns of the adenine mutants to a number of purines and purine derivatives.

Table 2. Growth of adenine mutants on purines and derivatives

\begin{tabular}{|c|c|c|c|c|c|c|c|c|c|c|c|}
\hline \multirow[b]{2}{*}{ Medium } & \multicolumn{11}{|c|}{ Locus } \\
\hline & ad-11 & $a d-10$ & $a d-9$ & $a d-8$ & $a d-6$ & $a d-5$ & $a d-4$ & $a d-3$ & $a d-2$ & $a d-1$ & Wild \\
\hline Complete & 14 & 13 & 16 & 21 & 8 & 19 & 8 & 11 & 19 & 19 & 19 \\
\hline Minimal & - & - & - & - & 2 & - & 2 & 2 & 3 & - & 20 \\
\hline + Adenine & 8 & 3 & 15 & 20 & 3 & 9 & 12 & 10 & 13 & 18 & 22 \\
\hline + Adenosine & 2 & - & 16 & 10 & - & - & 5 & 7 & 7 & - & 22 \\
\hline+ AMP & - & - & 10 & - & - & - & - & 2 & 7 & - & 22 \\
\hline+ Inosine & 3 & - & 13 & 12 & 2 & - & 8 & 8 & 12 & 2 & 20 \\
\hline+ Hypoxanthine & 8 & 2 & 19 & 18 & 4 & - & 9 & 9 & 13 & 5 & 20 \\
\hline guanine & - & - & 14 & - & - & - & - & - & - & - & - \\
\hline $\begin{array}{l}+ \text { Adenine and } \\
\text { guanosine }\end{array}$ & 12 & 2 & 15 & 20 & 7 & 9 & 10 & 9 & 14 & 20 & 21 \\
\hline guanine & - & - & - & - & - & 一 & - & 1 & - & - & - \\
\hline $\begin{array}{l}\text { + Adenosine an } \\
\text { guanosine }\end{array}$ & 5 & - & 13 & 9 & 3 & - & 4 & 8 & 12 & 3 & 17 \\
\hline
\end{tabular}

Entries appear as colony diameters (in $\mathrm{mm}$.) after $50 \mathrm{hr}$ growth. Strains used: wild type, BC9/66; ad-1, G 1912; ad-2, P 816; ad-3, M 8 and P 823; ad-4, P902; ad-5, GR 100.11 (derived from P 838); ad-6, P 809; ad-8, M6 and G1904; ad-9, MS 165 and JR 53; ad-10, GR712 (derived from G 2597); ad-11, M15.

All supplements used at a final concentration of $50 \mu \mathrm{g} . / \mathrm{ml}$.

Representative members of each locus were chosen for the determination of response to different degrees of adenine supplementation. After incubation for $50 \mathrm{hr}$ each strain exhibited its maximum growth on medium containing only $5 \mu \mathrm{g} . / \mathrm{ml}$. adenine-the maximum growth rates being essentially the same as those shown in Table 2. The highest concentration of adenine supplementation tested was $100 \mu \mathrm{g} . / \mathrm{ml}$.; there was no evidence of inhibition of growth at the higher concentrations after $50 \mathrm{hr}$ incubation. However, continued incubation to $125 \mathrm{hr}$ revealed that while the maximum growth rate was still attained on medium containing adenine, $5 \mu \mathrm{g} . / \mathrm{ml}$. inhibition of growth was evident in some cases at the highest concentrations. Strains BC6/65 (wild type), $a d-4, a d-5$ and $a d-9$ showed this effect; in each case the onset of inhibition was at adenine, $50 \mu \mathrm{g} . / \mathrm{ml}$. and at $100 \mu \mathrm{g} . / \mathrm{ml}$. the growth was about $85 \%$ of the maximum.

Of the extracts subjected to the chromatographic search for accumulation products only those of $a d-1$ and $a d-5$ showed definite accumulations. The mutants $a d-2, a d-3$, $a d-4$, and $a d-8$ showed no accumulation products distinct from wild type. Alleles of the $a d-6, a d-10$ and $a d-11$ loci were alike in showing very weak accumulations which were difficult to interpret.

The main accumulation product of $a d-1$ was a fast-running $\left(R_{F} 0 \cdot 35\right)$ compound staining bright yellow immediately on spraying with diazosulphonic acid, this colour fading to grey after spraying with sodium carbonate. This compound is very like that prod uced by the Neurospora 'adenine-purple' mutants of the ad-3 locus (Bernstein, 1961). The similarity goes further than this in that the raw extract of $a d-1$ was unique 
among the Coprinus extracts in being a completely opaque purple-brown colour, all others were transparent yellow-orange; an extract of Neurospora-ad-3 prepared as a control was also an opaque purple colour. Thus despite the lack of appreciable accumulation of pigment in the mycelium of Coprinus ad-1 during growth, probably because of the neutral $\mathrm{pH}$ value of Coprinus media, such an accumulation was very evident on extraction. It is likely that a change to acid $\mathrm{pH}$ values occurring during the extraction procedures allowed the accumulated imidazole to polymerise to the pigment. The fast running Coprinus ad-1 accumulant was examined spectroscopically; it had no peak of absorption in the ultraviolet region, but did show the broad end-absorption, starting at about $210 \mathrm{~m} \mu$, reported for 5-aminoimidazole ribotide (AIR) by Levenberg \& Buchanan (1957). The Bratton-Marshall reaction product (Bratton \& Marshall, 1939) of this substance was an orange-red chromophore which absorbed maximally at $500 \mathrm{~m} \mu$. These results were consistent with the identification of the major accumulant of Coprinus $a d-1$ as AIR. A slower-running $\left(R_{F} 0 \cdot 1\right)$ compound also detected in extracts of Coprinus ad-1 was not positively identified because of an excessive background of contaminating substances. When spectroscopically compared with wild-type eluate from the same chromatographic position (i.e. the wild-type eluate was used in the reference cuvette position) a distinct absorption peak was observable at $308 \mathrm{~m} \mu$, together with end-absorption from $220 \mathrm{~m} \mu$. Absorption at $305 \mathrm{~m} \mu$ is associated with the pigment produced by Neurospora-ad-3 (Bernstein, 1961), and is also associated with the raw extract of Coprinus ad-1. It is thus possible that the minor 'accumulant' was composed of some residual AIR (which would account for the end-absorption) which had been retained by the pigment (accounting for the $308 \mathrm{~m} \mu$ absorption peak).

Alleles of the ad-5 locus accumulated a compound which stained red after spraying with diazosulphonic acid; this densely coloured area faded to a cluster of grey spots after the sodium carbonate spray. This compound was tentatively identified as 5-amino-4-imidazole- $N$-succinocarboxamide ribotide (or riboside), SAICAR, from this colour reaction. The substance was eluted in acetate buffer (pH 5) and showed a very marked absorption peak at $268 \mathrm{~m} \mu$. Published data for SAICAR (Lukens \& Buchanan, 1959) gives 267-269 $\mathrm{m} \mu$ for peak absorption at pH 5. The BrattonMarshall reaction product of this accumulant absorbed maximally at $560 \mathrm{~m} \mu$; this is again characteristic of SAICAR and confirms the identification.

Alleles of the $a d-6, a d-10$ and $a d-11$ loci were alike in showing a very weak accumulation of a slow-running $\left(R_{F}\right.$ approximately 0.05$)$ substance. No isolation could be made from ad-6 extracts; $a d-10$ and $a d-11$ substances were isolated although the poor resolution lead to much contamination, mainly by compounds absorbing maximally at $260 \mathrm{~m} \mu$. The use of a wild-type reference abolished the 260 absorption and it became clear that eluates of both Coprinus $a d-10$ and ad-11 showed a weak absorption peak (not detectable in the wild type eluate) in the region of $298-310 \mathrm{~m} \mu$, coupled with broad end-absorption.

\section{DISCUSSION}

The information which can be obtained from growth tests of adenine mutants is limited by the fact that the majority of the intermediates in purine biosynthesis are rather exotic compounds; they are not readily available, and are not readily taken up by the cell. Probably the most useful fact arising from the studies with Coprinus lagopus 
mutants summarized in Table 2 is that ad-5 did not respond to hypoxanthine whereas all other loci did. This places the ad-5 'block' after the formation of inosinic acid (IMP), i.e. in one of the two reactions specific to adenosine-5'-phosphate (AMP) synthesis. The status of the other purines is confused by the non-uniformity of response. In some cases adenosine and AMP did not replace adenine, implying that the ribosyl compounds did not enter the organism and were not degraded extracellularly to the free base. On the other hand these two compounds did substitute for the adenine requirement of some mutants; whether this was the result of contamination by the free base or was a genuine genetic difference has not yet been determined. It may be significant that the mutants which responded only to the free base are assumed to be blocked after imidazole ring closure, while most of those which respond to adenosine and AMP are thought to be blocked in steps before closure of the ring.

With regard to the adenine/guanine inhibition it should be noted that ad-9 alone among the Coprinus mutants was completely free from any inhibition. The ad-9 mutation is possibly caused or accompanied by some change in the permeability of the cell membranes, or in the structure of a regulatory enzyme.

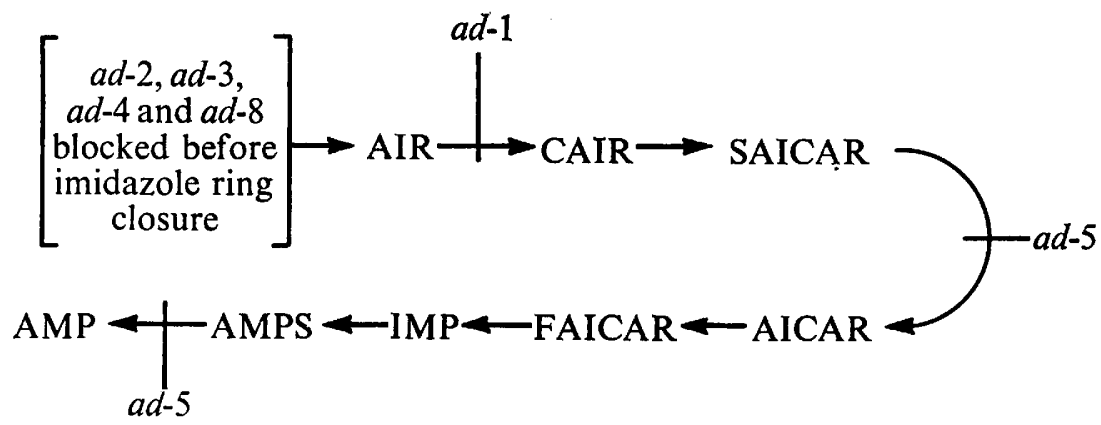

Fig. 2. Correlation of adenine mutants of Coprinus lagopus with steps in purine biosynthesis. Key: AIR, 5-aminoimidazole ribotide; CAIR, 5-amino-4-imidazole-carboxylic acid ribotide; SAICAR, 5-amino-4-imidazole- $N$-succino-carboxamide ribotide; AICAR, 5-amino-4imidazolecarboxamide ribotide; FAICAR, 5-formamido-4-imidazolecarboxamide ribotide; IMP, inosinic acid; AMPS, adenylosuccinic acid ribotide; AMP, adenosine-5'-phosphate.

By basing the conclusions on growth responses and the identities of accumulated aminoimidazoles some of the adenine loci can be correlated with steps in purine biosynthesis (Fig. 2). The $a d-2, a d-3, a d-4$ and $a d-8$ loci all responded to adenine or hypoxanthine and accumulated no imidazoles. They can be assigned to steps which precede closure of the imidazole ring. Accumulation of 5-aminoimidazole ribotide or riboside (AIR) by ad-1 indicates an inability to carboxylate AIR to 5-amino-4imidazolecarboxylic acid ribotide (CAIR). Since ad-5 did not respond to hypoxanthine, and yet accumulated 5-amino-4-imidazole- $N$-succinocarboxamide ribotide (SAICAR) it can be confidently assumed to lack the bifunctional enzyme 'adenylosuccinase'. Some alleles of the ad-5 locus not used in this investigation have been shown to lack adenylosuccinase activity (P. R. Day \& C. F. Roberts, personal communication). The ad-5 locus was termed adhi-1 by Cowan (cited in Casselton, 1965) because the growth rate of all ad-5 alleles was enhanced when histidine was provided as well as adenine. The data presented here suggest that the histidine 'requirement' is in fact the expression of a sparing reaction in that, if histidine biosynthesis is repressed by an 
exogeneous supply of the amino acid, there will be less call on what must be limited amounts of endogenous adenosine-5'-phosphate. The designation ad-5 should therefore be maintained. There is not sufficient evidence for any suggestion to be made about the positions of the $a d-6, a d-10$ and $a d-11$ mutations in the pathway.

I wish to thank Dr D. H. Morgan for his continued help and encouragement throughout the work. Facilities at the University of Hull were kindly provided by Professor N. F. Robertson, and at the John Innes Institute by Drs K. S. Dodds and J. R. S. Fincham. Drs A. Coddington and T. K. Sundaram of that Institute gave valuable advice on biochemical techniques. The work was performed during the tenure of a Science Research Council Research Studentship.

\section{REFERENCES}

Ames, B. N. \& Mrtchell, H. K. (1952). The chromatography of imidazoles. J. Am. chem. Soc. 74, 252.

BERNSTEIN, H. (1961). Imidazole compounds accumulated by purine mutants of Neurospora crassa. J. gen. Microbiol. $25,41$.

Bratton, A. C. \& MARShall, E. K. (1939). A new coupling component for sulphanilamide determination. J. biol. Chem. 128, 537.

CAsselton, L. A. (1965). The production and behaviour of diploids of Coprinus lagopus. Genet. Res. $6,190$.

DAY, P. R. (1959). A cytoplasmically controlled abnormality of the tetrads of Coprinus lagopus. Heredity, Lond. 13, 81.

DAY, P. R. (1962). Genetics of Coprinus lagopus: acrylamide gel electrophoresis used to detect enzymic differences. Rep. John Innes Instn. 53, 17.

Day, P. R. \& Anderson, G. E. (1961). Two linkage groups in Coprinus lagopus. Genet. Res. $2,414$.

Gollub, E. G. \& GoTs, J. S. (1959). Purine metabolism in Bacteria. VI. Accumulations by mutants lacking adenylosuccinase. J. Bact. 78, 320 .

LeVENBERG, B. \& BuchanAN, J. M. (1957). Biosynthesis of the purines. XII. Structure, enzymatic synthesis and metabolism of 5-aminoimidazole ribotide. J. biol. Chem. 224, 1005.

Lukens, L. N. \& Buchanan, J. M. (1959). Biosynthesis of the purines. XXIII. The enzymatic synthesis of $N$-(-5-amino-1-ribosyl-4-imidazolecarbonyl)-L-aspartic acid 5'-phosphate. J. biol. Chem. 234, 1791.

MagasaniK, B. \& Karibian, D. (1960). Purine nucleotide cycles and their metabolic role. J. biol. Chem. 235, 2672.

MOORE, D. (1966). The Formal Genetics of Coprinus lagopus (sensu Buller). Ph.D. Thesis, University of Hull. 